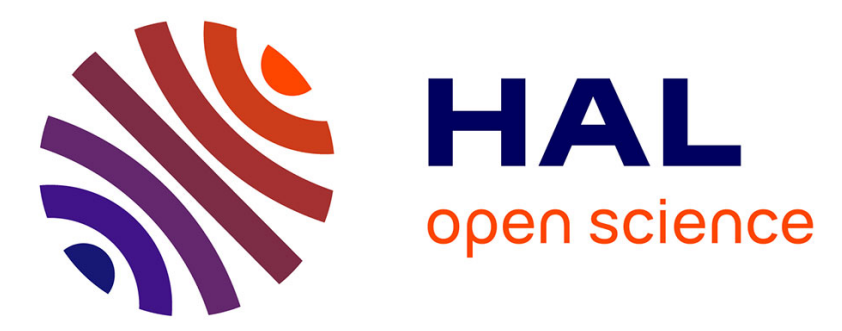

\title{
Le débat inachevé des historiens français sur les " lois mémorielles " et la pénalisation du négationnisme: retour sur une décennie de controverse
}

Boris Adjemian

\section{- To cite this version:}

Boris Adjemian. Le débat inachevé des historiens français sur les " lois mémorielles " et la pénalisation du négationnisme: retour sur une décennie de controverse. Revue arménienne des questions contemporaines, 2012. halshs-01510050

\section{HAL Id: halshs-01510050 \\ https://shs.hal.science/halshs-01510050}

Submitted on 18 Apr 2017

HAL is a multi-disciplinary open access archive for the deposit and dissemination of scientific research documents, whether they are published or not. The documents may come from teaching and research institutions in France or abroad, or from public or private research centers.
L'archive ouverte pluridisciplinaire HAL, est destinée au dépôt et à la diffusion de documents scientifiques de niveau recherche, publiés ou non, émanant des établissements d'enseignement et de recherche français ou étrangers, des laboratoires publics ou privés. 


\title{
Études arméniennes contemporaines
}

15 (2012)

Légiférer sur la contestation des génocides : débats et enjeux

Boris Adjemian

\section{Le débat inachevé des historiens français sur les « lois mémorielles » et la pénalisation du négationnisme : retour sur une décennie de controverse}

\begin{abstract}
Avertissement
Le contenu de ce site relève de la législation française sur la propriété intellectuelle et est la propriété exclusive de l'éditeur.

Les œuvres figurant sur ce site peuvent être consultées et reproduites sur un support papier ou numérique sous réserve qu'elles soient strictement réservées à un usage soit personnel, soit scientifique ou pédagogique excluant toute exploitation commerciale. La reproduction devra obligatoirement mentionner l'éditeur, le nom de la revue, l'auteur et la référence du document.

Toute autre reproduction est interdite sauf accord préalable de l'éditeur, en dehors des cas prévus par la législation en vigueur en France.
\end{abstract}

\section{revues.org}

Revues.org est un portail de revues en sciences humaines et sociales développé par le Cléo, Centre pour l'édition électronique ouverte (CNRS, EHESS, UP, UAPV).

Référence électronique

Boris Adjemian, «Le débat inachevé des historiens français sur les « lois mémorielles » et la pénalisation du négationnisme : retour sur une décennie de controverse », Études arméniennes contemporaines [En ligne],

15 | 2012, mis en ligne le 01 décembre 2014, consulté le 11 décembre 2014. URL : http://eac.revues.org/416

Éditeur : Union générale arménienne de bienfaisance

http://eac.revues.org

http://www.revues.org

Document accessible en ligne sur : http://eac.revues.org/416

Ce document est le fac-similé de l'édition papier.

Bibliothèque Nubar de I'UGAB 


\section{Le débat inachevé des historiens français sur les «lois mémorielles » et la pénalisation du négationnisme : retour sur une décennie de controverse}

BORIS ADJEMIAN Docteur en histoire (IRIS-EHESS)

es « Sages » du conseil constitutionnel ont-il
satisfaction aux historiens le 28 février der-
nier en censurant la loi dite Boyer, comme le
laissait entendre aussitôt le communiqué de l'association Liberté pour l'Histoire qui prenait acte de ce que, « en France, il ne revient pas au Parlement de légiférer sur l'histoire $\gg^{1}$ ? C'est l'impression qui semble dominer à l'intérieur d'un espace public où les prises de position les plus médiatisées ont été celles qui dénonçaient, depuis la fin décembre, le vote de cette loi « tendant à réprimer la contestation de l'existence de génocides reconnus par la loi » comme une atteinte à la liberté d'expression faisant peser le risque d'une « soviétisation de l'histoire $»^{2}$. Il serait pourtant caricatural de laisser croire que les historiens sont unanimes au sujet des questions que soulève la loi Boyer. Bien moins univoques en réalité qu’on a coutume de l'affirmer, leurs positions reflètent des différences épistémologiques au sein de leur discipline, des manières différentes de concevoir l'engagement du savant dans la cité, mais aussi des clivages politiques et idéologiques qui traversent la société française dans son ensemble et auxquels n'échappe pas la profession historienne. La pluralité de ces positions doit être exa-
1. Communiqué de l'association Liberté pour l'histoire, 29 février 2012.

2. Pierre Nora, « Lois mémorielles : pour en finir avec ce sport législatif purement français », Le Monde du 28 décembre 2011. 
minée à l'aune des controverses suscitées, depuis le milieu des années 2000, par la discussion ou l'adoption au Parlement de plusieurs textes de lois relatifs à des questions d'histoire et de mémoire demeurées politiquement ou socialement sensibles. Alors que chacun de ces textes aurait mérité d'être étudié dans sa singularité, leur qualification globale sous l'étiquette des «lois mémorielles » s'est rapidement imposée dans le débat public, sa simple évocation paraissant suffire à susciter, les années passant, des réactions de plus en plus épidermiques, non seulement chez une partie des historiens, mais aussi dans le monde politique et sous la plume de nombreux éditorialistes de la presse. Il est donc nécessaire pour expliquer les positions antagoniques au sein de la profession historienne, au terme de ce que nous pourrions appeler, avec un brin de provocation, la « décennie des "lois mémorielles" », de revenir au préalable sur les préconstruits de cette notion et sur les limites quelle a imposées au débat. Au-delà, il convient de ne pas minorer les difficultés que soulève l'adoption d'une législation destinée à contrer le négationnisme, en rappelant les craintes exprimées par des historiens, de la loi Gayssot (1990) à l'affaire Pétré-Grenouilleau (2005), de voir le travail du législateur aboutir à judiciariser le passé en figeant une ou des vérités historiques. Ces objections ne peuvent être balayées d'un revers de main. Elles méritent d'être prises au sérieux. Pour autant, bien que recevables, les critiques contre les dernières tentatives de pénalisation de la contestation des génocides ne permettent pas de répondre à cette question qui reste posée aux historiens : la société doit-elle ou non se prémunir - et si oui, comment - contre les falsifications de l'histoire et le négationnisme? 


\section{LE TOURNANT DE L'ANNÉE 2005 : UNE MOBILISATION DES HISTORIENS EN ORDRE DISPERSÉ}

Dans la chronologie des mobilisations historiennes qui ont émaillé la dernière décennie, l'année 2005 apparaît comme un tournant, avec l'adoption, le 23 février 2005, d'une loi « portant reconnaissance de la Nation et contribution nationale en faveur des Français rapatriés », dont l'article 4 préconisait que « les programmes scolaires reconnaissent en particulier le rôle positif de la présence française outre-mer, notamment en Afrique du Nord, et accordent à l'histoire et aux sacrifices des combattants de l'armée française issus de ces territoires la place éminente à laquelle ils ont droit ». C'est cette même année qu'éclate l'affaire PétréGrenouilleau, du nom de ce spécialiste de l'histoire des traites négrières contre lequel une plainte fut déposée en septembre par un collectif des « Antillais-Guyanais-Réunionnais », suite à une brève interview accordée au Journal du Dimanche du 12 juin 2005. C'est, enfin, le 12 décembre 2005 qu'a été rendue publique la désormais célèbre pétition «Liberté pour l'Histoire $\gg^{3}$, initialement signée par 19 personnalités ${ }^{4}$, qui demanda l'abrogation de « dispositions législatives indignes d'un régime démocratique ». Elle visait ainsi la loi du 13 juillet 1990 « tendant à réprimer tout acte raciste, antisémite ou xénophobe », dite loi Gayssot, qui punit la contestation de l'existence des crimes contre l'humanité définis comme tels par le tribunal militaire international de Nuremberg en 1945 ; la loi du 29 janvier 2001 « relative à la reconnaissance du génocide arménien de

3. Pétition qui a donné lieu à la création d'une association du même nom, actuellement présidée par Pierre Nora.

4. Les premiers signataires étaient Jean-Pierre Azéma, Elisabeth Badinter, Jean-Jacques Becker, Françoise Chandernagor, Alain Decaux, Marc Ferro, Jacques Julliard, Jean Leclant, Pierre Milza, Pierre Nora, Mona Ozouf, Jean-Claude Perrot, Antoine Prost, René Rémond, Maurice Vaisse, Jean-Pierre Vernant, Paul Veyne, Pierre Vidal-Naquet et Michel Winock. 1915 »; la loi du 21 mai 2001 « tendant à la reconnaissance de la traite et de l'esclavage en tant que crime contre l'humanité », dite loi Taubira; enfin, la loi évoquée plus haut du 23 février 2005, dite loi Mekachera. Les signataires fondaient leur demande sur un rappel appuyé de ce qui différencie l'histoire de la mémoire, des sensibilités du temps présent, de la morale et du dogme. Ils accusaient ces quatre lois d'avoir « restreint la liberté de l'historien » et de lui avoir « dit, sous peine de sanctions $\gg$, ce qu'il devait chercher et ce qu'il devait trouver. La 
pétition Liberté pour l'Histoire réunit, dès les premières semaines qui suivirent sa publication, plus d'un millier de signatures, dont celles de nombreux historiens et professeurs du secondaire

5. Le président de la République, Jacques Chirac, venait de confier le 9 décembre au président de l'Assemblée nationale, Jean-Louis Debré, l'organisation d'une commission chargée « d'évaluer l'action du Parlement dans le domaine de l'Histoire et de la mémoinonce, le 4 janvier 2006, en faveur d'une réécriture de l'article incriminé de la loi du 23 février 2005. L'alinéa litigieux fut abrogé par décret le 15 février 2006.

6. Les premiers signataires en étaient les chercheurs et universitaires Claude Liauzu, Gilbert Meynier, Gérard Noiriel, Frédéric Régent, Trinh Van Thao et Lucette Valensi.

7. Les initiateurs de la pétition, comme Claude Liauzu, devaient revenir rapidement sur cette demande d'abrogation totale et ne plus réclamer que la réécriture de son article 4, le reste du texte némettant pas de jugement de valeur particulier sur le fait colonial et n'invitant quà la reconnaissance des souffrances des victimes des guerres d'indépendance en Afrique du Nord et au développement de la recherche dans ce domaine. Voir Pierre Boilley, « Loi du 23 février 2005, colonisation, indigènes, victimisations. Évocations binaires, représentations primaires », Politique africaine, 98, juin 2005, pp. 131-140. re », avant que celle-ci ne se pro-

enseignant l'histoire.

Afin de bien mesurer la portée de cet appel du 12 décembre 2005, il importe cependant de revenir quelques mois en arrière et de prendre en considération d'autres actions également initiées par des historiens. Les 19 pétitionnaires se sont en effet déclarés à contretemps de la séquence politique ouverte par le vote de l'article 4 de la loi du 23 février 2005, alors même que, la polémique faisant rage depuis plusieurs mois entre les promoteurs de la loi Mekachera et ses opposants, elle semblait sur le point de trouver son dénouement ${ }^{5}$. Depuis le mois de mars, l'adoption de ce texte avait suscité une vague de protestations de la part de spécialistes de l'histoire coloniale, d'universitaires et d'enseignants du secondaire. Une pétition lancée à l'initiative de l'historien Claude Liauzu et de plusieurs de ses collègues, 6 , parue dans Le Monde du 25 mars 2005 sous le titre « Non à l'enseignement d'une histoire officielle », avait été soutenue et relayée par la Ligue des droits de l'homme et plusieurs syndicats d'enseignants. Ces historiens, qui faisaient valoir les implications que la loi Mekachera avait sur l'exercice de leur métier et sur les aspects pédagogiques, scientifiques et civiques de leur discipline, réclamaient son abrogation urgente ${ }^{7}$ au motif qu'elle imposait une histoire officielle contraire à la neutralité scolaire et au respect de la liberté de pensée, en demandant que les programmes de l'Éducation nationale reconnaissent « le rôle positif de la présence française outre-mer », c'est-à-dire de la colonisation. Ils constataient qu' « en ne retenant que le "rôle positif" de la colonisation », cette loi imposait « un mensonge officiel sur des crimes, sur des massacres allant parfois jusqu'au génocide, sur l'esclavage, sur le racisme hérité de ce passé », bref sur toutes les heures obscures de 
la colonisation et les idéologies les moins glorieuses qui avaient soustendu l'entreprise coloniale et la domination exercée sur les peuples colonisés. Les signataires de la pétition du 25 mars mettaient enfin en garde leurs concitoyens contre les effets prévisibles de l'adoption d'un texte flattant le « communautarisme nationaliste » au risque de susciter « en réaction le communautarisme de groupes ainsi interdits de tout passé $\gg^{8}$. C'est dans la foulée de cette première mobilisation d'historiens qu'a été créé le Comité de vigilance face aux usages publics de l'histoire (CVUH), au printemps 2005.

Dans son Manifeste, s'il reconnaît que les acteurs de la vie publique sont fondés à « puiser dans l'histoire des arguments pour justifier leurs causes ou leurs intérêts », le CVUH s'élève contre toute forme d'instrumentalisation du passé et souligne la nécessité d'une action collective de la part de « tous ceux qui refusent que l'histoire soit livrée en pâture aux entrepreneurs de mémoire », dans un contexte où « l'information-spectacle et l'obsession de l'audimat poussent constamment à la surenchère, valorisant les provocateurs et les amuseurs publics, au détriment des historiens qui ont réalisé des recherches approfondies, prenant en compte la

8. Le texte peut ici être lu comme faisant référence au controversé et critiqué « Appel pour des Assises de l'anticolonialisme postcolonial »du 16 janvier 2005, mieux connu sous le titre de la pétition « Nous sommes les indigènes de la République ! » complexité du réel ». Rappelant les tentatives précédentes des négationnistes de « travestir l'histoire de la Shoah pour servir les thèses de l'extrême droite », le CVUH constate que la question coloniale est désormais devenue elle aussi un enjeu majeur de manipulation et d'instrumentalisation du passé. Il dit son inquiétude face à une loi du 23 février 2005 « sous-tendue par une vision conservatrice du passé colonial », qui traduit « le profond mépris du pouvoir à l'égard des peuples colonisés et du travail des historiens » et qui illustre la propension « du pouvoir politique et des médias [...] à imposer des jugements de valeur au détriment de l'analyse critique des phénomènes » historiques. Réfutant toute tentation de considérer les historiens « comme des experts qui détiendraient la Vérité sur le passé », il affirme que le rôle des chercheurs et des enseignants en histoire est « de mettre à la disposition de tous les connaissances et les questionnements susceptibles de favoriser une meilleures compréhension de l'histoire, de manière à nourrir l'esprit critique des citoyens, tout en leur fournissant des éléments qui leur permettront d'enrichir leur propre jugement politique, au lieu de parler à leur place $\gg$. 
9. «Manifeste du Comité de Vigilance face aux usages publics de l'histoire », 17 juin 2005, http://cvuh.blogspot.fr/2007/0 $2 /$ manifeste-du-comite-devigilance-face.html, mis en ligne le 6 février 2007.

10. Outre la pétition du 25 mars 2005, la première en date contre ce texte, il faut signaler la motion adoptée à Paris par le Comité national de l'Association des professeurs d'histoire et de géographie (APHG), le 22 mai 2005, contre l'article 4 de la loi Mekachera, demandant « que soit mis fin aux pratiques qui consistent à instrumentaliser l'enseignement de l'histoire au service des "devoirs de mémoire" » et rappelant « que les contenus d'enseignement en histoire et en géographie doivent se fonder sur les acquis de la recherche scientifique, pour laquelle l'Université et le CNRS doivent être dotés de moyens suffisants ». Début décembre 2005 enfin, suite au refus des députés de la majorité d'abroger l'article 4 tant controversé, une nouvelle pétition était lancée à l'initiative de la Ligue des droits de l'homme et d'un collectif d'historiens, intitulée « Nous n'appliquerons pas l'article 4 de la loi du 23 février stipulant que "les programmes scolaires reconnaissent le rôle positif" de la colonisation », qui devait recueillir en un mois 1120 signatures dont 572 d'historiens enseignants et chercheurs. Elle dénonçait une « loi de régression culturelle en ce début de XXI ${ }^{\mathrm{e}}$ siècle », « imposée
C'est donc une posture modeste que les membres du CVUH ont souhaité adopter en se positionnant dans le débat non comme des censeurs ou des autorités, mais, soucieux de ne pas s'enfermer « dans une tour d'ivoire », comme des citoyens désireux d'alerter et d'éclairer leurs concitoyens sur les usages politiques (au sens large) du passée

En comparaison avec l'écho relatif des pétitions contre la loi du 23 février 2005 qui l'avaient précédée ${ }^{10}$, le large retentissement de l'appel du 12 décembre 2005 appelant à abroger indistinctement les « lois mémorielles », qui a été perçu ou présenté comme reflétant la position des historiens dans leur ensemble, en dit long « sur la façon dont fonctionnent les relations de pouvoir dans le monde intellectuel français $\gg^{11}$. La protestation des historiens aurait sans doute conservé la forme prise depuis le printemps, celle d'une mobilisation de chercheurs et d'enseignants du secondaire et de l'université, si la dimension politique du débat n'avait pris un tour nouveau, avec l'entrée en scène du président de la République ${ }^{12}$. C'est alors, semble-t-il, que le débat qui faisait rage depuis des mois a subitement paru s'incarner dans la figure des 19 premiers signataires de l'appel Liberté pour l'Histoire - des personnalités célèbres qui ont immédiatement été présentées dans les médias comme les porte-parole des historiens ${ }^{13}$.

L'appel des « 19 » peut donc difficilement être tenu pour l'étalon d'une mobilisation des historiens qui fut bien plus précoce, en France, dans les mois qui le précédèrent. Surtout, il se différenciait des prises de position antérieures en ce qu'il réclamait l'abrogation indistincte de quatre lois, toutes jugées également contraires à la démocratie, et non du seul article 4 de la loi Mekachera. Il établissait de fait une équivalence entre, d'une part, une loi célébrant le rôle prétendu14 ment positif de la colonisation et, d'autre part, des textes qui reconnais- 
saient solennellement, au nom de la Nation, un crime contre l'humanité (l'esclavage et la traite négrière), un génocide (l'extermination des Arméniens), ou encore qui pénalisaient la négation du génocide commis contre les Juifs pendant la Seconde Guerre mondiale. C'est parce qu'ils étaient en désaccord avec cette position jusqu'au-boutiste que de nombreux historiens ont signifié leur refus de s'associer à la pétition Liberté pour l'Histoire, dont seuls 2 des 19 premiers signataires sétaient par ailleurs associés à la pétition du 25 mars, comme l'observait l'historien Marcel Dorigny. Ce dernier regrettait un « amalgame choquant », tout en faisant remarquer à la rédaction du magazine L'Histoire, qui l'avait diffusé, que l'appel des $\ll 19 »$ ne faisait pas l'unanimité des historiens ${ }^{14}$. L'appel du 12 décembre 2005 a ainsi été presque aussitôt suivi de mises au point et d'interpellations de la part d'historiens qui ne se reconnaissaient pas dans cette démarche et en contestaient le bienfondé : «Il ne nous paraît pas possible que des historiens puissent, au nom de la "liberté de l'histoire", s'affranchir des normes, aujourd'hui reconnues par les plus hautes instances internationales, qui font de la condamnation et, encore plus, de la prévention des génocides et des crimes contre l'humanité une impérieuse nécessité. [...] Par ailleurs, la "liberté de l'histoire" ne se limite nullement à la question du rapport à la loi. Nous devons nous interroger aussi sur les relations que les historiens entretiennent avec le journalisme, avec le monde associatif et la classe politique. [...] Ce débat ne doit pas être monopolisé par quelques personnalités académiques ou médiatiques car personne n'est habilité dans ce pays à parler au nom de tous les historiens $\gg^{15}$. Peu après l'annonce par Jacques Chirac, le 4 janvier 2006, que l'article 4 de la loi du 23 février 2005 serait réécrit, un nouvel appel d'historiens regrettait « l'amalgame » fait par les signataires de Liberté pour par des groupes de pression nostalgiques du colonialisme et revanchards, nourris d'une culture d'extrême droite », et demandait « aux institutions universitaires, aux IUFM, aux associations professionnelles, aux syndicats d'enseignants, aux parents d'élèves d'organiser un vaste mouvement de protestation » contre cette « loi scélérate ». Voir http://www.ldh-toulon.net/spip.php?article1058, dernière consultation $1^{\text {er }}$ octobre 2012.

11. Gérard Noiriel et Nicolas Offenstadt, « Les historiens et les autres. Sur le rôle des historiens dans les débats publics récents en France. Une lecture », http:// cvuh.blogspot.fr/2007/03/leshistoriens-et-les-autres-sur-le. html, mis en ligne le 8 mars 2007. Dernière consultation $1^{\text {er }}$ octobre 2012.

12. Soucieux d'apaiser les esprits alors que la contestation avait gagné les élus et habitants des DOM-TOM, et de désensabler sa majorité de droite au Parlement qui ne pouvait se résoudre à perdre la face, Jacques Chirac déclarait publiquement, le 9 décembre 2005 : « Dans la République, il n'y a pas d'histoire officielle. Ce n'est pas à la loi d'écrire l'histoire. L'écriture de l'histoire, c'est l'affaire des historiens ». Le président de la République préparait simultanément les esprits à l'enterrement du fameux article 4, en confiant une commission à ce sujet au président de l'Assemblée nationale (voir note 5).

13. Lire le texte de Gérard Noiriel, « De l'histoire-mémoire 
aux "lois mémorielles". Note sur les usages publics de l'histoire en France », dans ce même dossier.

14. Marcel Dorigny, « Lettre adressée à la rédaction de la revue L'Histoire (2 janvier 2006) et restée inédite ». La lettre ne fut pas publiée par L'Histoire mais est consultable sur http://cvuh. blogspot.fr/search?q=dorigny. Voir aussi la « Lettre adressée le 14 décembre 2005 à un signataire de l'appel des 19 historiens », par Bruno Belhoste, sur http://cvuh. blogspot.fr/2007/03/lettreadressee-le-14-decembre-2005un.html. Textes également cités par Gérard Noiriel et Nicolas Offenstadt dans le dossier « Histoire et politique autour d'un débat et de certains usages » de la revue Nouvelles FondationS, 2006/2 - n 2, pp. 65-75.

15. « Appel pour une vigilance sur les usages publics de l'histoire », par Michel Giraud, Gérard Noiriel, Nicolas Offenstadt et Michèle Riot-Sarcey, http:// cvuh.blogspot.fr/2007/03/appel -pour-une-vigilance-sur-lesusages.html, mis en ligne le 9 mars 2007.

16. « Urgence : l'abrogation de la loi du 23 février 2005 contre l'indépendance de l'histoire », 4 janvier 2006, http://www.ldhtoulon.net/spip.php?article1119. Les signataires, dont une majorité d'historiens, étaient Hélène d'Almeida-Topor, Jean Baubérot, Esther Benbassa, Alban Bensa, Jean-Pierre Chrétien, Fanny Colonna, Alice Conklin, Myriam Cottias, Georges Dupon-Lahitte,
l'Histoire « entre des lois qui, toutes posent problème aux historiens, qui risquent de peser sur leur liberté, mais qui n'ont ni le même contenu, ni les mêmes finalités », au risque d'instaurer « la confusion » et « de provoquer des incompréhensions dans l'opinion ». Tout en rappelant avec force la nécessité de préserver l'indépendance de l'historien, cet appel soulignait que « les historiens professionnels ne sont pas les seuls à être concernés par le passé, et [qu']ils n'en ont pas la propriété $\gg^{16}$.

Les prises de position relatives à l'une ou l'autre de ces quatre lois, en cette année 2005, révélaient donc des points de vue pour le moins divergents et l'absence patente de consensus au sein de la profession historienne. Malgré tout, l'appel « Liberté pour l'Histoire » est vite apparu comme celui de l'ensemble des historiens, en raison de la notoriété de ses initiateurs, mais aussi peut-être du caractère sans nuance de ses exigences qui se prêtait bien à un exposé simplificateur de la controverse. C'est dans ce contexte que la notion de « lois mémorielles », utilisée en boucle dans la sphère médiatique et politique, et reprise au vol par les partisans de leur abrogation dans leurs multiples interventions publiques, s'est imposée dans les termes du débat. Si les généralisations et les raccourcis sémantiques sont commodes et ne surprennent guère dans une logique médiatique, leur utilisation par des historiens professionnels censés éclairer leurs concitoyens mérite réflexion.

LA CONSTRUCTION D'UN ÉPOUVANTAIL : LA NOTION DE « LOIS MÉMORIELLES » ET SES USAGES

Dans un livre d'entretiens avec le philosophe François Azouvi qu'il publia en 2006, l'un des initiateurs de la pétition Liberté pour l'Histoire devenu peu 6 après le premier président de l'association du même nom, l'académicien 
René Rémond, faisait continuellement référence, sans jamais en proposer une définition, à la notion de « lois mémorielles ${ }^{17}$. Que l'objet de cet ouvrage ait explicitement été de se livrer à une « rétrospective des lois mémorielles ${ }^{18}$ ne paraissait étrangement pas justifier la moindre précaution de langage. Un tel usage était significatif de la place soudainement acquise par cette expression au tournant des années 2005 et 2006, dans le débat sur les rapports que devaient ou pas entretenir la loi et le passé, comme si le sens de la notion qu'elle recouvrait était désormais connu et reconnu de tous. La notion de « loi mémorielle » n'en demeurait pas moins obscure. Son utilisation était (et demeure aujourd'hui) loin d'être neutre. Comme le notait plus récemment le rapport de la mission d'information de l'Assemblée nationale « sur les questions mémorielles » signé par Bernard Accoyer, « le débat public, depuis quelques années, s'est focalisé sur les lois dites "mémorielles", un qualificatif surtout employé par leurs détracteurs. [...] Le concept de "loi mémorielle" est très récent puisque l'expression n'apparaît qu'en 2005 pour désigner rétrospectivement un ensemble de textes dont le plus ancien ne remonte qu'à $1990 \gg^{19}$. Constat qui n'empêche d'ailleurs pas le rapport d'user de cette notion sans donner plus de précision : même s'il donne raison à Robert Badinter, qui a déclaré lors d'une audition que la loi Gayssot n'est pas une loi mémorielle ${ }^{20}$, on reste bien en peine, à la lecture de ce rapport, de savoir ce qu'est une loi mémorielle ${ }^{21}$. De fait, il semble que la volonté de ne traiter de chacune des lois considérées qu'au travers de l'entité conceptuelle « lois mémorielles » traduit des préoccupations de nature politique face au danger qu'elles représenteraient pour

Marcel Dorigny, Mohammed Harbi, Jean Jamin, Claude Liauzu, Patricia Lorcin, Gilles Manceron, Gilbert Meynier, Pap Ndiaye, Gérard Noiriel, JeanMarc Régnault, Frédéric Régent, Michèle Riot-Sarcey, Jean-Pierre Sainton et Patrick Weil.

17. François Azouvi et René Rémond, Quand l'État se mêle de l'Histoire, Paris, Stock, 2006. Ce livre reprenait l'argumentation déjà développée dans René Rémond, « L'Histoire et la Loi », Études, 2006/6, pp. 763 773. Pour un compte-rendu critique, voir Boris Adjemian, «Quelques questions sur les "lois mémorielles" et la demande de leur abrogation », http://cvuh. blogspot.fr/2007/11/quelquesquestions-sur-les-lois.html.

18. F. Azouvi et R. Rémond, op. cit., 2006, p. 33.

19. Bernard Accoyer, Rassembler la Nation autour d'une mémoire partagée, Rapport d'information de la mission d'information sur les questions mémorielles, Paris, Assemblée nationale, novembre 2008, p. 11.

20. Id., 2008, pp. 26, 46.

21. L'argumentation du rapport tend à suggérer que le « caractère mémoriel » des articles d'une loi est avéré lorsque ceux-ci ont un caractère déclaratif, et non pas normatif. Id., 2008, p. 32. l'unité nationale et le pacte républicain. Ce que le rapporteur de la mission d'information sur les questions mémorielles dit attendre d'une législation relative à des faits du passé n'est rien d'autre, en effet, que le « renforcement du sentiment d'appartenance collective des 
citoyens à la nation », un « objectif de réconciliation nationale » supposé être « au cœur du devoir de mémoire ». Le rapport cherche ainsi « les clefs d'une politique rassemblant la nation autour d'une mémoire partagée », dressant le constat que, « en effet, les questions mémorielles dépassent largement la seule problématique des lois dites "mémorielles", car celle-ci ne fait que refléter un certain malaise dans les rapports qu'entretiennent les Français avec leur "roman national" $\gg^{22}$.

Les prises de position historiennes les plus médiatisées ne sont pas toujours exemptes de ces considérations politiques. Sous la plume des plus éminents représentants de l'association Liberté pour l'Histoire, le combat contre les « lois mémorielles » apparaît comme un sursaut face à la « repentance » et contre toute critique qui vien-

22. Id., 2008, p. 67, 91 .

23. Pierre Nora, « Malaise dans l'identité historique », initialement publié dans la revue $L e$ Débat, 2006/4, nº 141, pp. 44-48, également reproduit dans P. Nora et F. Chandernagor, Liberté pour l'histoire, Paris, CNRS Éditions, 2008.

24. B. Accoyer, op. cit., 2008, p. 210-211. drait lézarder le précieux édifice du roman national en mettant en lumière des « communautés » ou des mémoires qui sont supposées lui être étrangères. La présentation faite par Pierre Nora ne laisse guère de doute à ce sujet : « Deux mille ans de culpabilité chrétienne relayée par les droits de l'homme se sont réinvestis, au nom de la défense des individus, dans la mise en accusation et la disqualification radicale de la France. Et l'école publique s'est engouffrée dans la brèche avec d'autant plus d'ardeur qu’à la faveur du multiculturalisme elle a trouvé dans cette repentance et ce masochisme national une nouvelle mission. Après avoir été le vaisseau pilote de l'humanité, la France est devenue ainsi l'avant garde de la mauvaise conscience universelle $\gg^{23}$. Ajoutant, devant la mission d'information de l'Assemblée nationale, que « cette révision générale de l'Histoire en fonction de la victime est extraordinairement dangereuse », non seulement au point de vue critique et intellectuel, mais aussi en ce qui concerne « l'intérêt national », et que seuls des historiens de gauche peuvent ne pas adhérer à cette idée ${ }^{24}$. Il s'inscrit pleinement, ce faisant, dans le propos de son prédécesseur René Rémond contre « des lois mémorielles qui tendent à ériger une mémoire particulière dictée ou imposée par une faction en vérité historique pour la communauté nationale ou pour l'humanité ». Elles sont jugées illégitimes au motif qu'elles ne résulteraient que de l'action de segments d'une société française de plus en plus travaillée par 
convenu de nommer le « communautarisme » disqualifie par avance toute initiative parlementaire sur de telles matières, comme par exemple la loi de 2001 sur le génocide arménien et la proposition de loi de 2006 sur la pénalisation de sa contestation ${ }^{25}$, qui ne traduiraient que l'opportunisme de députés succombant au travail de sape des lobbies et aux sirènes de l'électoralisme (compte tenu des fameux « cinq cent mille »- quand ils ne vont pas jusqu'au « demi-million » $-\mathrm{d}^{\prime} \ll$ Arméniens » de France, dont l'existence politique ne repose sur rien de tangible mais qui, enfants compris, et quelque puissent être par ailleurs leurs opinions personnelles, sont censés voter comme un seul homme et toujours répondre présents à ce genre de sollicitations intéressées). La « multiplication des lois mémorielles » représente pour ses détracteurs, comme le synthétise le rapport Accoyer en se rangeant à cet avis, « un risque de fragilisation de la société française ». En effet, « la qualification du passé national à l'aune de concepts juridiques qui criminalisent notre histoire aura nécessairement des conséquences sur la façon dont les Français perçoivent leur pays ». En outre, ajoute le rapporteur, « dans leur ensemble, les questions mémorielles qui font débat depuis plus de trente ans dans notre pays, que ce soit la colonisation et l'esclavage, la résurgence de l'histoire de la collaboration ou le rapport à la guerre d'Algérie, dressent une image de notre passé qui n'est pas toujours "heureuse" et qui peut affaiblir le sentiment de fierté nationale. De fait,

25. Il s'agit de la proposition de loi déposée par le député Didier Migaud au nom du groupe socialiste, le 12 avril 2006, visant à punir des mêmes peines que celles prévues par la loi Gayssot les personnes qui auraient contesté publiquement l'existence du génocide de 1915. Les sénateurs refusèrent toutefois par deux fois d'inscrire ce texte, adopté par l'Assemblée nationale en première lecture le 12 octobre 2006, à l'ordre du jour du Sénat.

26. B. Accoyer, op. cit., 2008, p. 54 .

27. Id., 2008, pp. 80-81. les lois mémorielles ne trouvent pas toujours un écho favorable au sein de la population française. Une partie de nos concitoyen peut même y voir une forme de repentance irritante, voire exaspérante $\gg^{26}$. La peur de lois qui divisent était manifeste dans l'argumentation développée par René Rémond en 2006, qui s'inquiétait, à travers l'évocation de la loi de 2001 sur le génocide arménien, de « l'action d'une minorité qui entend faire reprendre par la nation entière sa mémoire particulière $\gg^{27}$. De ce point de vue, le rejet de la loi Taubira est tout aussi éclairant, car il est explicitement mis en relation avec la crainte de voir les revendications se multiplier au sein des populations « issues de l'immigration », particulièrement des nouvelles générations qui pourraient mettre en cause le passé colonial de la France. L'hostilité vis-à-vis des deux lois votées en 2001, 
notamment la loi Taubira, ne s'inscrit donc pas seulement dans une démarche intellectuelle mais aussi, dimension qu'on ne peut éluder, dans le combat très politique mené ces dernières années par voie de presse et de pamphlets contre une supposée tendance de la France héritière des Lumières à « l'auto-flagellation ». Dans cette perspective, la mise en exergue de mémoires jusqualors négligées par le roman national, ou d'histoires auxquelles on dénie leur place dans l'histoire de la France, comme celles de l'immigration, suscite de farouches oppositions, comme l'ont illustré récemment les polémiques sur l'introduction de quelques

28. Lire Laurence de Cock, Suzanne Citron et Jean-Pierre l'histoire de France, il parait que C dans l'air du temps », http://cvuh.blogspot.fr/2010/0 9/virer-lafrique-de-lhistoire-defrance.html, mis en ligne le 24 novembre 2010. Jean-Pierre Chrétien, « À propos du texte de Benoît Rayski, diffusé sur le site Atlantico, "De Vercingétorix à Napoléon : dans les poubelles de l'histoire" », http://cvuh. blogspot.fr/2011/09/propos-dutexte-de-benoit-rayski.html, mis en ligne le 8 septembre 2011.

29. C'est le point de vue défendu par exemple par Françoise Chandernagor face à Christiane Taubira dans le Nouvel Observateur du 23 février 2006. Chrétien, «Virer l'Afrique de

heures d'études sur des civilisations africaines et asiatiques dans les programmes d'histoire des classes de $6^{\mathrm{e}}$ et de $5^{\mathrm{e}}$ au collège ${ }^{28}$.

La loi du 21 mai 2001 « tendant à la reconnaissance de la traite et de l'esclavage en tant que crime contre l'humanité » s'est vue accorder une place de choix dans la dénonciation des « lois mémorielles ». Elle est souvent visée par ceux qui s'émeuvent d'une mise en accusation de la France, laquelle serait sommée de se confronter aux pages sombres de son passé et de faire le jeu d'une « repentance » jugée inacceptable, pour des raisons politiques. La condamnation de la traite esclavagiste transatlantique, c'est-à-dire celle menée entre des ports européens et des colonies européennes du Nouveau Monde, gêne parce qu'elle ne s'accompagne pas d'une condamnation tout aussi forte de l'esclavage en Afrique et des traites négrières musulmanes à destination des marchés du Moyen-Orient et de l'océan Indien ${ }^{29}$. La dénonciation de la traite comme un crime contre l'humanité est implicitement perçue comme une condamnation de l'Occident, de son histoire et de ses héritages. Peu importe que les mouvements abolitionnistes aient été très actifs dès le $\mathrm{XVIII}^{\mathrm{e}}$ siècle, particulièrement en Angleterre, mais aussi en France. Pour que la condamnation de la traite soit acceptable, il faudrait donc en quelque sorte faire preuve d'équilibrisme, et ne pas s'en prendre à la traite européenne sans condamner avec autant de vigueur les traites extra-européennes. Ainsi, en pleine controverse sur le rôle prétendument positif de la colonisation, le renvoi dos à dos de la loi Mekachera, qui légitimait 
l'œuvre coloniale de la France, et de la loi Taubira, peut difficilement être interprété comme une comparaison objective dénuée d'arrière-pensées idéologiques et politiques : « l'amendement controversé sur les aspects positifs de la colonisation est en fait la riposte directe à cette loi [Taubira]. Celle-ci, en déclarant crime contre l'humanité l'esclavage, emportait la condamnation du fait colonial. Ces deux textes sont symétriques et solidaires. Le premier ne considère que les aspects négatifs, le second met l'accent sur les aspects positifs. C'est la raison pour laquelle je pense qu'ils doivent avoir le même sort : n'abroger que l'un serait un choix purement politique, pour ou contre la colonisation $\gg^{30}$. La demande d'abrogation des deux lois ne procédait donc pas seulement du souci de préserver l'indépendance des historiens face au politique, mais aussi d'empêcher que l'on prît position pour ou (surtout ?) contre le colonialisme : «La France doit-elle avoir honte de son passé colonial ? La colonisation n'aurait-elle apporté que des malheurs ? » ${ }^{31}$ Était-ce vraiment là le débat ? On a peine à croire, d'ailleurs, que cette position exprimée par le président de Liberté pour l'Histoire de l'époque, reflétait fidèlement l'opinion de chacun des 19 premiers signataires de l'appel du 12 décembre 2005, ne serait-ce que celle du regretté Pierre VidalNaquet (décédé en 2006), bien connu pour son com-

30. F. Azouvi et R. Rémond, op. cit., 2006, p. 34-36.

31. Id., 2006, p. 52-53.

32. Id., 2006, p. 81.

33. Id., 2006, p. 82-83. bat contre la torture pendant la guerre d'Algérie.

Quelles que soient les arrière-pensées qui président - ou non - à son usage, la notion de « lois mémorielles » conduit à confondre sous le même sceau de l'illégitimité des textes de lois pourtant fort dissemblables dans leurs motivations et dans leur portée. Les « lois mémorielles » sont devenues, pour leurs détracteurs, le symbole de la faiblesse des politiques face aux pressions de communautés qui entendent contraindre la nation à légiférer à leur avantage, c'est-à-dire au bénéfice de « mémoires singulières $\gg^{32}$, dans un contexte historique et politique qui privilégie «les identités communautaires [...], la constitution des identités particulières, qu'elles soient ethniques, politiques ou sexuelles $\gg^{33}$. Les lois de 2001 sur le génocide arménien et sur la traite et l'esclavage, ainsi que la loi Gayssot de 1990 dans une certaine mesure, deviennent, dans cette lecture à charge des « lois mémorielles » qui leur dénie toute portée universaliste, la simple expression d'égoïsmes communautaires allant ouvrir la boite de Pandore des revendications particularistes les plus déplacées. C'est, en 
définitive, une lecture purement communautariste de la société et de l'action du Parlement que porte la notion de « lois mémorielles » : « le Parlement a jugé bon de qualifier les massacres d'Arméniens : pourquoi ne pas étendre la condamnation à d'autres atrocités ? À propos de la traite négrière il sagit de donner satisfaction aux Antillais. Ce sera bientôt le tour des arabo-musulmans $[s i c]$. Et le Parlement sera sommé de condamner les Croisades $\gg^{34}$. Si le Parlement souhaite légiférer aujourd'hui, avec la loi Boyer, sur des génocides étrangers à la France,

34. Id., 2006, pp. 41-42.

35. Pierre Nora, « Liberté pour l'histoire ! », Le Monde du 11 octobre 2008. Voir aussi, du même, « Gare à la criminalisation générale du passé ! », Le Figaro du 17 mai 2006, et « L'histoire, la mémoire et la loi en France (1990-2008) », communication présentée lors du $\mathrm{XX}^{e}$ congrès des sciences historiques, Amsterdam, 23 août 2010, http://www.lphasso.fr/index.php?option $=\mathrm{com}_{-}$ content $\&$ view $=$ article $\& i d=159$ \%3Apierre-nora-qlhistoire-lamemoire-et-la-loi-en-france$1990-2008$ q \& c a t i d = $53 \% 3$ Aactualites\&Itemid $=170 \&$ lang=fr, mis en ligne le 14 janvier 2011. Voir également, entre autres, Jacques Julliard, « Les poisons de la mémoire », Le Nouvel Observateur du 22 décembre 2005. Françoise Chandernagor, «Lois mémorielles : un monstre législatif », Le Figaro du 29 décembre 2011. F. Azouvi et R. Rémond, op. cit., 2006, p. 32. alors pourquoi pas sur les Indiens d'Amérique ou la famine provoquée par le pouvoir stalinien en Ukraine en 1932-1933 ? « À quand la Vendée ? À quand la Saint-Barthélemy ? À quand les albigeois, les cathares », s'interrogent en cœur les initiateurs de Liberté pour l'Histoire ${ }^{35}$. On peut certes comprendre que la perspective d'une multiplication sans fin de lois qui porteraient un jugement ou une appréciation sur tel ou tel événement du passé inquiète les parlementaires, et c'est précisément de cette crainte quest née la mission d'information de l'Assemblée nationale dirigée en 2008 par Bernard Accoyer. Mais cet argument, agité par une partie des historiens qui ne semblent pas toujours réaliser que les bûchers de Monségur ou le massacre de la Saint-Barthélemy ne sont la cible d'aucune entreprise connue de négationnisme, n’apporte pas une réponse convaincante à la question que posait la loi Boyer : le citoyen doit-il, ou non, être protégé contre la contestation des génocides ?

La question avait déjà été abordée par deux propositions de lois - rejetées - de pénalisation de la négation du génocide des Arméniens, en 2004 et 2006. Le rapport de la mission d'information sur les questions mémorielles mettait en garde en 2008 contre le risque qu'il y aurait à légiférer sur l'histoire en recourant à des catégories juridiques comme le crime contre l'humanité ou le génocide, au risque d'une « inflation des actions en responsabilité civile de l'historien et de porter atteinte à sa liberté d'opinion et d'expression ». Il faisait valoir, par ailleurs, qu'en 22 dehors de la loi Gayssot qui ne saurait prévenir que la négation des crimes 
visés par le tribunal de Nuremberg, le droit pénal français réprime déjà l'incitation à la haine raciale et à la discrimination ${ }^{36}$. Sans relever, toutefois, que l'actuelle législation était impuissante à juguler la contestation de l'existence d'autres génocides que ceux commis par les nazis. Au-delà de la récente adoption de la loi Boyer par l'Assemblée nationale et par le Sénat, puis de son rejet par le conseil constitutionnel, la question de l'opportunité d'un arsenal juridique spécifique reste donc posée aux historiens, qui sont aussi des citoyens. Pas plus que du reste de la société française, elle n'apporte de leur part de réponse unanime.

\section{LA VOIE ÉTROITE DES HISTORIENS : ENTRE UN PASSÉ JUDICIARISÉ ET LES ASSASSINS DE LA MÉMOIRE}

La séquence politique qui s'est ouverte avec la discussion et l'adoption de la loi Boyer à l'Assemblée nationale, à la fin décembre 2011, puis au Sénat à la fin janvier 2012, avant de se refermer par la saisine du Conseil constitutionnel et la censure finale de la loi, à la fin février, a été de nouveau présentée comme la résurgence de la polémique déjà ancienne sur les « lois mémorielles ». Si les réserves ou la franche opposition face à ce texte semblent avoir été relativement mieux partagées parmi les historiens qu'elles ne l'avaient été face aux lois dont l'existence avait, dans les années précédentes, suscité la controverse au sein de la profession $^{37}$, la loi Boyer a au moins eu le mérite de poser la question du négationnisme. C'est en tâchant de répondre à cette question majeure, et non en se cantonnant au rejet indistinct des « lois mémorielles », que la loi visant à réprimer la contestation des génocides mériterait d'être

36. B. Accoyer, op. cit., 2008, pp. 50, 94-96.

37. Lire par exemple la position du CVUH, qui avait refusé de s'associer à la demande d'abrogation de la loi de 2001 mais qui a exprimé son opposition au vote de la loi présentée par Valérie Boyer devant l'Assemblée nationale, critiquant un texte qui relevait d'une instrumentalisation de l'histoire pour des motifs idéologiques et électoralistes : http:// cvuh.blogspot.fr/search/label/g \%C3\%A9nocide\%20arm\%C3\% A9nien, mis en ligne le 30 décembre 2011.

38. Communiqué de presse de Pierre Nora au nom de Liberté pour l'Histoire, 24 janvier 2012, http://www.lph-asso.fr/ index.php?option=com_content \&view $=$ article\&id $=171 \% 3$ Acom munique-de-presse-de-pierrenora-au-nom-de-liberte-pourlhistoire\&catid $=5 \% 3$ Acommuni ques\&Itemid = 15\&lang $=\mathrm{fr}$, consulté le 5 octobre 2012. discutée.

Déplorant l'adoption du texte au Sénat, l'association Liberté pour l'Histoire reprenait l'argumentation qu'elle avait développée contre les « lois mémorielles » depuis 2005 en affirmant que, « sur le plan national », la loi Boyer représentait un danger car elle alimentait et instituait « ce qu'il faut bien appeler une guerre civile des mémoires ${ }^{38}$. Nous avons vu plus haut que cet argument rejoignait le combat de certains cou- 
rants d'opinion contre la « repentance » de la France face aux revendications et à la propension au « victimisme » de « communautés » imparfaitement assimilées. Ces demandes de reconnaissance officielle de la part de mémoires blessées et longtemps ignorées ne sont pas perçues comme légitimes parce qu'elles sont considérées comme émanant, en quelque sorte, de pièces rapportées de l'histoire nationale et de la société française. Dénoncer la guerre civile des mémoires qu'encouragerait une loi contre la contestation des génocides tient donc davantage de l'opinion politique que d'une « objectivité » pourtant érigée en vertu cardinale de l'historien par les contempteurs des « lois mémorielles ». Elle n'est absolument pas neutre. Mais les autres arguments invoqués contre la loi Boyer ne peuvent être écartés d'un revers de main et participent d'un vrai débat entre les historiens. Dans le même communiqué, l'as-

39. Ibid.

40. Pierre Nora, « Lois mémorielles : pour en finir avec ce sport législatif purement français », Le Monde du 28 décembre 2011. sociation Liberté pour l'Histoire dénonçait les inconvénients et les absurdités de cette loi « sur le plan historique » et démocratique, déclarant que, dans le cas où elle serait effectivement appliquée, elle interdirait « toute recherche historique approfondie sur une des premières grandes tragédies du XX $\mathrm{X}^{\mathrm{e}}$ siècle », et qu'elle contrevenait « à la Déclaration des droits de l'homme et du citoyen en bafouant la liberté d'expression $\gg{ }^{39}$. On retrouve ici une des critiques qui ont été le plus souvent formulées contre les « lois mémorielle » par les porte-parole de Liberté pour l'Histoire : la loi de 2001 reconnaissant le génocide arménien (il est vrai, purement déclarative) et la loi dite Taubira déclarant l'esclavage et la traite comme un crime contre l'humanité portent sur des événements d'un passé lointain dont les acteurs, fussent-ils coupables, ne peuvent plus rendre compte de leurs actes. Les premières victimes désignées de ces lois seraient, dès lors, les historiens et la recherche historique. Raison pour laquelle Pierre Nora, qui rappelait ce qu'il considérait comme le fâcheux précédent de la loi Gayssot, parlait récemment du risque de « soviétisation de l'histoire $»^{40}$.

Les premières critiques contre la loi Gayssot, adoptée en juillet 1990 sous le coup de « la profonde émotion provoquée par la négation du génocide hitlérien et par les conséquences de la banalisation du nazisme », ont été soulevées par l'historienne Madeleine Rebérioux, dans un texte souvent cité par ceux qui dénoncent aujourd'hui les « lois mémorielles ». Elle faisait valoir que l'arsenal juridique préexistant à la loi 
Gayssot avait déjà permis de faire condamner au civil en 1981 le négationniste Robert Faurisson, pour diffamation publique ${ }^{41}$, ceci - point essentiel - sans que les tribunaux aient eu à se prononcer sur la «vérité historique » : «Non seulement rien n'est plus difficile à constituer en délit qu'un mensonge historique, mais le concept même de vérité historique récuse l'autorité étatique. [...] Les génocides peuvent et doivent être "pensés", comparés et, dans la mesure du possible, expliqués. Les mots doivent être pesés, les erreurs de mémoire rectifiées. Expliquer le crime, lui donner sa dimension historique, comparer le génocide nazi à d'autres crimes contre l'humanité, c'est le combattre. C'est ainsi - et non par la répression - que l'on forme des esprits libres ${ }^{42}$. Il faut toutefois souligner que cette prise de position initiale ne s'inscrit pas dans le même contexte idéologique que celui de la controverse qui a divisé les historiens au cours des années 2000. Les réserves de Madeleine Rebérioux, ainsi que celles exprimées à l'époque par Pierre VidalNaquet, qui avait méthodiquement démonté luimême le mensonge négationniste sans éprouver la nécessité de recourir aux tribunaux ${ }^{43}$, sont restées isolées. À l'exception de ces deux grandes voix, les historiens français n'ont pas sérieusement remis en cause le bienfondé de la loi Gayssot. C'est plus tard, et davantage au sujet du génocide des Arméniens ou de la qualification de la traite négrière en tant que crime contre

41. R. Faurisson avait déclaré sur une antenne de radio que « le prétendu gazage et le prétendu génocide juif ne sont qu’un seul et même mensonge historique qui a permis une gigantesque escroquerie politico-financière dont les principaux bénéficiaires sont l'État d'Israël et le sionisme international ».

42. Madeleine Rebérioux, «Le génocide, le juge et l'historien », L'Histoire, $\mathrm{n}^{\circ} 138$, novembre 1990.

43. Pierre Vidal-Naquet, Les assassins de la mémoire. "Un Eichmann de papier » et autres essais sur le révisionnisme, Paris, $\mathrm{La}$ Découverte, 1991 [1987]. l'humanité, que les historiens se sont véritablement inquiétés des effets en retour de la loi sur leur liberté d'expression. Quelques « affaires » retentissantes ont servi de catalyseur.

La plus récente, l'affaire Pétré-Grenouilleau, a provoqué une forte mobilisation après le dépôt d'une plainte contre les propos tenus par cet historien en septembre 2005, mobilisation large dans laquelle les signataires de Liberté pour l'Histoire ne furent pas les premiers. Il s'agissait de défendre la liberté académique d'un collègue spécialiste de l'histoire des traites négrières qui restait dans son domaine de compétence lorsqu'il discutait de l'utilisation du terme de génocide, face à une plainte qui était sans doute excessive, quelque soit la maladresse des propos tenus ${ }^{44}$, et qui fut d'ailleurs retirée par les plaignants au début de l’année 2006. On doit 
44. Il faut d'ailleurs souligner que, contrairement à ce qui est souvent avancé par les détracteurs des « lois mémorielles », par exemple Marc Ferro devant la mission d'information parlementaire de 2008, les propos de cet historien ne tombaient nullement « sous le coup de la loi "Taubira" » et « d'une vérité officielle nouvelle » quelle aurait instaurée (cf. B. Accoyer, op. cit., 2008 , p. 218, 221), la loi déclarant seulement que l'esclavage et la traite furent des crimes contre l'humanité.

45. Pierre Nora, « Gare à la criminalisation générale du passé », Le Figaro du 17 mai 2006. Voir aussi le point de vue de Pierre Vidal-Naquet dans Le Monde du 3 février 1999, «Sur le négationnisme imaginaire de Gilles Veinstein ». Sans doute l'accusation de « négationnisme » à son endroit était-elle excessive et contreproductive, comme le faisait justement observer Michel Cahen dans une tribune intitulée : « Négationnisme : ne nous trompons pas », Libération du 31 décembre 1998. Dans un article qui plaide en faveur d'un approfondissement des savoirs sur le génocide de 1915, Vincent Duclerc remarque aussi que ce type de mise en accusation peut accroittre la difficulté de travaux sur la destruction des Arméniens, « dans la mesure où le doute méthodique et la critique des sources qui caractérisent la pratique de l'historien pourraient être brutalement assimilés à des comportements négationnistes », peut-être lier les réactions suscitées par l'affaire PétréGrenouilleau aux plaies laissées par l'affaire Veinstein, du nom de cet historien ottomaniste dont l'élection au Collège de France fut fortement perturbée en 1998, suite à un article qu'il avait publié trois ans plus tôt sur le génocide de 1915 dans le magazine L'Histoire et qui lui avait valu d’être accusé de négationnisme. L’affaire, si elle ne fut pas portée devant les tribunaux, fut perçue par une partie des historiens comme la marque d'une forme de «terrorisme intellectuel $»^{45}$. Mais il faut remonter quelques années plus tôt, à l'affaire Lewis, pour trouver l'exemple d'une procédure judiciaire qui soit allée jusquà son terme après avoir été lancée contre un historien pour des propos tenus publiquement. L'historien américain Bernard Lewis fut en effet condamné par le tribunal de grande instance de Paris, le 21 juin 1995, à verser un franc de dommages et intérêts, ainsi que dix mille francs au Forum des associations arméniennes de France et quatre mille à la Ligue internationale contre le racisme et l'antisémitisme (LICRA), qui le poursuivaient devant cette juridiction civile ${ }^{46}$ pour des propos tenus deux ans plus tôt dans un grand quotidien français. Au cours d'une interview, cet historien orientaliste avait réfuté le qualificatif de « génocide » concernant les déportations et massacres de 1915, qu'il considérait comme « la version arménienne de cette histoire » : «si l'on parle de génocide, cela implique qu'il y ait eu politique délibérée, une décision d'anéantir systématiquement la nation arménienne. Cela est fort douteux. Des documents turcs prouvent une volonté de déportation, pas d'extermination $»^{47}$. Certes, comme le faisait observer Madeleine Rebérioux dans une manière de suite à son précédent article sur la loi Gayssot, Bernard Lewis n'avait pas formellement contesté qu'il y ait eu des « tueries » et des souffrances de part et d'autre. Dès lors les historiens pouvaient s'inquiéter de ce que l'un 
de leurs collègues, professeur à Princeton, soit mis en cause devant la justice pour avoir simplement exprimé son opinion : « l'essentiel, à mes yeux, ne réside pas dans l'exacte qualification qu'il convient d'apporter à ces tueries que Lewis ne nie pas, mais bien dans le choix de faire appel à un tribunal pour trancher, et dans le jugement rendu ${ }^{48}$. Quels quaient été les mérites intellectuels de la position défendue à l'époque par Madeleine Rebérioux, celle-ci revenait à ignorer que l'argumentation développée par Bernard Lewis, sous couvert de l'objectivité de l'historien, n'était en fait qu'une redite fidèle de la thèse officielle turque pour expliquer la disparition des Arméniens de l'Empire ottoman comme un effet regrettable des désordres et des animosités réciproques créés par la guerre : « Nul doute que des choses terribles ont eu lieu, que de nombreux Arméniens - et aussi des Turcs - ont péri. Mais on ne connaîtra sans doute jamais les circonstances précises et les bilans des victimes ${ }^{49}$. C'était, surtout, faire abstraction que, s'agissant des crimes de masses en général, l'enjeu de la qualification est crucial. Dans le cas arménien, le refus de reconnaître le caractère organisé et planifié des déportations et massacres par le gouvernement ottoman dirigé par les Jeunes Turcs, et donc de lui reconnaître le caractère du crime de génocide, est constitutif de la négation érigée en politique officielle par l'État turc. Arc-bouté sur la relativisation du caractère des massacres, la dilution des responsabilités et la minoration du nombre des pertes, ce négationnisme avait déjà une longue pratique et, disons-le, une longue histoire derrière lui au moment de l'affaire Lewis. $\mathrm{Ne}$ pas en tenir compte, dans la dénonciation actuelle des limites opposées à la liberté des historiens, c'est s'empêcher de comprendre, non seulement les revendications « mémorielles » du temps présent, mais aussi l'enjeu d'un débat sur l'opportunité d'une législation pour réprimer la contestation des génocides. Il est tout à fait conduisant du même coup à un rejet du savoir potentiellement apporté par les turcologues, alors que « les formes d'adhésion au discours officiel doivent être analysées avant d'être stigmatisées ». Voir Vincent Duclerc, « Les historiens et la destruction des Arméniens », Vingtième siècle. Revue d'histoire, 81, janvier-mars 2004, pp. 137-153, (cf. pp. 143144). L'article de Gilles Veinstein n'en reprenait pas moins, malheureusement, des arguments constitutifs de la négation institutionnalisée en Turquie depuis des décennies, se fondant au passage sur l'ouvrage de Kâmuran Gürün, Le dossier arménien (1984), qui fait sourire les spécialistes et dont le moins qu'on puisse dire est qu'il ne répond pas vraiment aux exigences minimales de la critique historique. Voir Gilles Veinstein, « Trois questions sur un massacre », L'Histoire, no 187 , avril 1995, pp. 40-41. L'attitude la plus cohérente, et la plus juste peutêtre, eût été de refuser de participer à la rédaction du dossier sur le « massacre des Arméniens » publié par L'Histoire, sachant que l'exposé du génocide serait mis en balance, « thèse » contre « thèse », avec un article dont l'auteur s'évertuait - en toute bonne foi probablement - à demander des preuves supplémentaires, à la demande d'ailleurs du magazine qui avait souhaité lui attribuer ce rôle du « discutant ».

46. Une plainte déposée au pénal dans les mêmes circonstances avait en effet été rejetée, les juges 
estimant que la loi Gayssot, invoquée par les plaignants, ne pouvait faire l'objet d'une interprétation extensive et servir à réprimer la contestation d'autres crimes que ceux reconnus par le Tribunal international de Nuremberg en 1945. C'est finalement sur la base de l'article 1382 du code civil que Bernard Lewis fut condamné à verser des dommages aux plaignants, pour sêtrre exprimé « sans nuance » sur un sujet sensible en ayant « occulté les éléments contraires à sa thèse » et avoir, à cette occasion, tenu des propos « fautifs » susceptibles de « raviver injustement la douleur de la communauté arménienne ».

47. « Un entretien avec Bernard Lewis », propos recueillis par Jean-Pierre Langellier et JeanPierre Peroncel-Hugoz, Le Monde du 16 novembre 1993. Ces affirmations furent critiquées dans un texte collectif signé par 35 personnes, dont André Chouraqui, Jacques Ellul, Alain Finkielkraut, André Kaspi, Yves Ternon et Jean-Pierre Vernant, et paru dans Le Monde du 27 novembre 1993 sous le titre « Cela s'appelle un génocide ». En réponse, Bernard Lewis fit une mise au point, dans les mêmes colonnes, le $1^{\text {er }}$ janvier 1994, en concluant : « il n'existe aucune preuve sérieuse d'une décision et d'un plan du gouvernement ottoman visant à exterminer la nation arménienne ».

48. Madeleine Rebérioux, «Les Arméniens, le juge et l'historien », L'Histoire, $\mathrm{n}^{\circ} 192$, octobre 1995. légitime de défendre l'idée selon laquelle Bernard Lewis n'avait pas mérité de se trouver devant un tribunal, et encore moins, a fortiori, d'être condamné, mais on aurait tort de croire que ses propos étaient anodins. Les défenseurs les plus idéalistes de la liberté d'expression seraient bien inspirés d'y regarder à deux fois avant d'en faire l'un des martyrs de leur cause.

Une énième contribution à la controverse, l'« Appel de Blois » de 2008, est emblématique des positions de principe qui, au nom d'une absolue liberté, tendent à considérer la répression du négationnisme comme une déclaration de guerre aux historiens. On touche ici au cœur du débat sur la liberté d'expression et ses limites, une question d'ailleurs classique pour les collégiens en cours d'éducation civique. Lancé à grand renfort de publicité dans le cadre des annuels Rendez-vous de l'Histoire par l'actuel président de l'association Liberté pour l'Histoire, l'appel dit de Blois mettait en garde historiens et politiques en Europe tant contre une « moralisation rétrospective de l'histoire », dans un contexte de « mémoires concurrentes », que contre la « censure intellectuelle » et la remise en question de la liberté, dans un État où l'autorité politique entreprendrait de « définir la vérité historique et de restreindre la liberté de l'historien sous la menace de sanctions pénales $»^{50}$. Or il se voulait une sorte de réponse à la proposition de décisioncadre des ministres de la justice de l'Union européenne adoptée le 19 avril 2007, concernant « la lutte contre certaines formes et manifestations de racisme et de xénophobie au moyen du droit pénal », et qui encourage la répression, dans tous les États membres, de l'apologie, la négation ou la banalisation des crimes de génocide, crimes contre l'humanité et crimes de guerre. Il pouvait donc paraitre pour le moins étrange de qualifier de liberticide un texte qui ne vise pas précisément à l'étatisation de l'histoire et reconnaît expressément les droits 
fondamentaux, au premier rang desquels la liberté d'expression, tels que définis par la Convention européenne des droits de l'homme ${ }^{51}$. Certes, les dérives dénoncées par l'appel de Blois ne sont pas imaginaires. Mais la comparaison de la situation créée en France par des textes comme la loi Boyer avec celle connue dans la Russie de Vladimir Poutine, où l'État se livre à une réhabilitation en règle du stalinisme, l'imposant jusque dans les manuels scolaires, pourra paraître disproportionnée ${ }^{52}$. Comme le faisait remarquer récemment le directeur du Center for Holocaust and Genocide Studies de l'université du Minnesota, il est du reste curieux que certains des historiens qui dénoncent la loi Boyer de la manière la plus véhémente n'aient pas eu un mot, dans leurs nombreuses prises de position publiques, au sujet $\mathrm{du}$ négationnisme officiel de l'État turc et des pressions qu'il est à même d'exercer, par le biais de différents relais, sur des chercheurs étrangers ${ }^{53}$.

Les spécificités du déni propres à chaque crime de masse posent une question à laquelle la dénonciation radicale de la loi Gayssot, présentée comme « la mère de toutes les lois mémorielles $\gg^{54}$, ne suffit pas à répondre. Pierre Vidal-Naquet l'avait rejetée car il jugeait qu'elle ouvrirait la voie à d'autres lois, portant sur d'autres crimes, qui constitueraient autant de dérapages législatifs. Il estimait que les historiens n'avaient rien à gagner à cette loi, et la société non plus, qui légitimerait ainsi les négationnistes dans leur posture de martyrs de la liberté d'expression. Mais les spécialistes de l'étude du génocide des Arméniens et de celui des Tutsi du Rwanda savent bien que leur négation n'est pas le fait de groupuscules politiques extrémistes et des voix marginales de « la petite bande abjecte » que dénonçait Pierre Vidal-Naquet lorsqu'il parlait des

49. «Un entretien avec Bernard Lewis », propos recueillis par Jean-Pierre Langellier et JeanPierre Peroncel-Hugoz, Le Monde du 16 novembre 1993.

50. http://www.lph-asso.fr/ index.php?option=com_content \&view $=$ article\&id $=47 \&$ Itemid $=$ 14\&lang=fr. Il a été signé par de nombreux historiens, dont certains n'avaient pourtant pas soutenu l'appel du 12 février 2005 qui exigeait l'abrogation des « lois mémorielles ».

51. Comme le faisaient remarquer les historiens Catherine Coquery-Vidrovitch, Gilles Manceron et Gérard Noiriel dans «Les historiens n'ont pas le monopole de la mémoire », Le Monde du 7 novembre 2008.

52. Lire Nicolay Kopozov, « Le débat russe sur les lois mémorielles », sur le site de Liberté pour l'Histoire : http://www.lphasso.fr/index.php?option $=$ com content $\&$ view $=$ article $\& i d=73 \& \mathrm{I}$ temid=181\&lang=fr, mis en ligne le 5 juillet 2009.

53. Bruno Chaouat, « Lois mémorielles : l'indignation sélective de Pierre Nora », Le Monde du 30 décembre 2011.

54. F. Azouvi et R. Rémond, op. cit., 2006, p. 17. « assassins de la mémoire ». En France, il n'est devenu commun d'entendre parler, sans précautions oratoires ou périphrases (« tragédie de 1915 », « massacre des Arméniens », « injustice faite au peuple arménien », etc.) du génocide de 1915 dans les médias et au plus haut niveau 
de l'État que depuis les années 2000, particulièrement après la commémoration, plus médiatisée qu’à l'habitude, du quatre-vingt-dixième anniversaire des événements. Donc après les votes de 1998 et de 2001 à l'Assemblée nationale et au Sénat ${ }^{55}$, dont il n'est pas

55. Sur les circonstances de ces votes et des débats au Parlement sur l'opportunité d'une reconnaissance du génocide des Arméniens passant par la loi, lire Olivier Masseret, « La reconnaissance par le parlement français du génocide arménien de 1915 », Vingtième Siècle. Revue d'histoire, 73, janvier-mars 2002, pp. 139-155. 56. C'est ce qui est arrivé à l'historien Jean-Pierre Chrétien et au journaliste Jean-François Dupaquier, poursuivis pour diffamation par l'ancien ambassadeur de France au Rwanda pour la période 1990-1994, dont ils avaient critiqué le recours à la thèse du « double génocide » (entre Hutu et Tutsi) comme un argument habituel des négationnistes du génocide des Tutsi de 1994. Voir la pétition de soutien signée en leur faveur par les chercheurs du Centre d'études des mondes africains et de la revue Politique africaine, notamment sur http:// www.ldh-france.org/ IMG/pdf/ es_chercheurs.pdf, mis en ligne le 25 octobre 2011.

57. Tribunal Permanent des Peuples, Le Crime de silence. Le génocide des Arméniens, Paris, Flammarion, 1984, p. 15. Également dans P. Vidal-Naquet, op. cit., 1991, p. 61. Pour_la_liberte_d_expression_d

interdit de penser qu'ils ont contribué, par l'expression publique d'une reconnaissance officielle de la France, à l'ancrer dans les esprits - et à rendre sa négation plus dérangeante. Quant à la reconnaissance explicite du génocide des Tutsi de 1994, qui a immédiatement été perçue dans certains milieux comme une mise en cause de la France, elle reste encore, dans notre pays, un enjeu politique brûlant et un processus inachevé, la négation pouvant être professée publiquement aussi simplement qu'une opinion, alors que les historiens du génocide peuvent être, eux, visés par des plaintes s'ils se risquent à caractériser la dialectique négationniste de tel ou tel ${ }^{56}$. La critique méthodique du discours et de l'imposture négationnistes par des spécialistes, certes nécessaire, est rendue beaucoup moins efficace par ce rapport de force particulier. Il suffit de rappeler les lignes, souvent citées - par des spécialistes du génocide arménien il est vrai -, du même Pierre VidalNaquet, pour faire entendre la difficulté qu'il y aurait à laisser le combat contre un négationnisme institutionnel aux seuls hommes de bonne volonté et à la seule rigueur de la méthode historienne : «Imaginons Faurisson ministre, Faurisson président de la République, Faurisson ambassadeur, Faurisson président de la commission historique turque, membre du Sénat de l'université d'Istanbul, Faurisson membre influent des Nations-Unies, Faurisson répondant dans la presse chaque fois qu'il est question du génocide des Juifs. Bref un Faurisson d'État doublé d'un Faurisson international et, avec tout cela, Talaat-Himmler jouissant depuis 1943 d'un mausolée solennel dans la capitale $\gg^{57}$.

Après avoir exigé l'abrogation de la loi de 2001 par laquelle la France reconnaissait publiquement le génocide des Arméniens de 1915, et en 
avoir critiqué le caractère communautariste, électoraliste, avoir maintes fois souligné que les faits auxquels elle se rapportait ne concernaient pas la France et les Français, que l'on ne pouvait enfin les qualifier de génocide au risque de l'anachronisme, l'association Liberté pour l'Histoire a, plus récemment, par deux fois au moins, invoqué ce nouvel argument : toute loi relative au génocide arménien en France, qu'il s'agisse de sa reconnaissance ou d'une pénalisation de sa négation, serait contreproductive pour la recherche et pour le dialogue arméno-turc. Sitôt après l'adoption de la loi Boyer au Sénat, elle regrettait « que cette loi aboutisse au contraire de ce que ses motifs allèguent : en poussant le nationalisme turc aux réactions les plus excessives, elle encourage le permanent négationnisme d'État ; elle complique en même temps le courageux combat pour l'indispensable reconnaissance des crimes du passé que mènent des milliers de citoyens et d'intellectuels turcs. Un combat dont Liberté pour l'histoire se déclare totalement solidaire ${ }^{58}$. Un mois plus tard, prenant acte avec satisfaction du rejet de la loi par le Conseil constitutionnel, l'association en profitait pour « redire la nécessité urgente d'engager le gouvernement turc à favoriser la mise en place d'une commission internationale d'historiens, sous l'égide, par exemple, de l'Unesco, chargée de faire, dans des conditions scientifiques, toute la lumière sur les tragiques événements de 1915 et le massacre des Arméniens. En

58. Communiqué de presse de Pierre Nora, au nom de Liberté pour l'Histoire, le 24 janvier 2012.

59. Communiqué de Liberté pour l'Histoire, 29 février 2012.

60. F. Azouvi et R. Rémond, op. cit., 2006, pp. 30-31, 79-80.

61. B. Accoyer, op. cit., 2008, p. 211. toute liberté pour l'histoire $\gg{ }^{59}$. Cette position, qui se calque sur la proposition d'une commission historique mixte faite par le gouvernement turc au gouvernement arménien en 2009, est finalement assez proche de celle que René Rémond défendait, au moment de l'appel du 12 décembre 2005 et de le la demande d'abrogation inconditionnelle des « lois mémorielles », lorsqu'il affirmait qu'en reconnaissant le génocide des Arméniens, « le Parlement tranchait un débat sur lequel les historiens sont partagés », alors que « nous ne disposons pas de la preuve décisive que le massacre des Arméniens a bien été un génocide $\gg^{60}$. Dans son audition par les membres de la mission parlementaire, Pierre Nora s'étonnait ainsi que, après qu'il ait « voulu organiser à Sciences-Po une rencontre entre historiens turcs et arméniens », ces derniers aient refusé, emportés qu'ils étaient par leur passion, leur intransigeance et le refus de discuter « avec les héritiers des criminels! ${ }^{61}$ 
Il faut expliquer ici pourquoi ces propos sont difficilement recevables, non point au regard des descendants de victimes ou des associations communautaires arméniennes qui pourraient prendre la « mouche » (pour parler comme Pierre Nora), mais vis-à-vis des chercheurs spécialistes de l'étude du génocide de 1915. Le débat serait certainement plus serein si l'opposition à la loi Boyer ou aux textes précédemment évoqués, opinion parfaitement respectable, ne s'accompagnait pas de temps à autre de la mise en doute des acquis de la recherche par des historiens non spécialistes qui font preuve d'une grande légèreté lorsqu'ils se prononcent sur ces questions historiques des plus sensibles. Un collectif d'historiens « spécialistes du génocide des Arméniens engagés depuis de nombreuses années dans la recherche et la publication d'ouvrages sur ce sujet $\gg$ exprimait ainsi, le 25 janvier dernier, son « complet désaccord avec les déclarations publiques tendant à faire de la réalité du génocide des Arméniens une thèse à discuter ou l'objet de quelque commission bipartite TurquieArménie devant statuer sur son existence », qu'il qualifiait de « manœuvre de diversion ». Tenant à rappeler solennellement que « toute opinion ne vaut pas démonstration », ces chercheurs soulignaient que « la matérialité et l'intention du génocide commis contre les Arméniens de l'Empire ottoman durant la Première Guerre mondiale ont été amplement démontrées par des historiens qui ont pris la peine de travailler sur ce sujet et savent de quoi ils parlent quand ils associent le concept de génocide au cas arménien, à la différence des auteurs de ces déclarations approximatives, forts d'une notoriété acquise sur d'autres sujets mais dont aucun n’a mis son nez dans les archives ou publié sur le sujet ». Le texte de ce collectif est certes passé plus ou moins inaperçu au milieu d'une controverse déjà très nourrie. On remarquera qu'il ne prenait aucunement position sur l'opportunité qu'il y avait ou non à légiférer sur la question des génocides - il fut d'ailleurs publié au lendemain seulement du vote de la loi Boyer au Sénat - et ne mettait en avant que des arguments scientifiques, arguant que « la nécessaire poursuite des travaux sur cet événement ne peut s'appuyer sur l'occultation ou le refus perpétuels des acquis de la recherche, par l'invocation d'une liberté scientifique que le négationnisme détourne et pervertit ». À la lecture de cette tribune, on peut ainsi penser que c'est sans doute, davantage que par une loi de pénalisation des négationnismes, grâce au soutien des institutions publiques à la recherche, qu’une des questions majeures posée par le 
récent débat pourrait trouver une solution : « Plus que jamais, la recherche sur les génocides avec une dimension comparatiste mérite une place plus importante en France, à l'université, à l'école, dans le monde de l'édition, au service du savoir et de la formation des citoyens. Le soutien à la recherche dans ce domaine devrait être une priorité de la République. Nous lançons aussi un appel aux pouvoirs publics en France pour la création d'une chaire d'histoire sur l'étude des génocides. Elle permettrait de former, d'éduquer et d'en finir avec les propos négationnistes $\gg^{62}$. Une telle politique de recherche permettrait de ne pas laisser la société démunie face aux négationnismes, sans pour autant passer par le vote de lois qui seront toujours suspectées d'instrumentalisation ou d'arrière-pensées, et critiquées en conséquence. Elle désamorcerait par avance, en les rendant vaines, les éventuelles tentatives de récupération politiciennes dont la campagne présidentielle de 2012 a donné la caricature, au détriment d'un débat sérieux et au mépris de l'intelligence des citoyens. Il n'est pas interdit de penser, pour finir, quelle trouverait un accueil consensuel chez les historiens.

62. Annette Becker, Hamit Bozarslan, Vincent Duclert, Raymond Kévorkian, Claire Mouradian et Yves Ternon,

Les feux de ce débat décennal sur les appréciations que le Parlement doit, ou ne doit pas, porter sur le passé, provisoirement éteints depuis la censure de la loi Boyer, ne manqueront sans doute pas de repartir à la « Appel pour l'Histoire du génocide des Arméniens », Libération du 25 janvier 2012.

63. Voir l'article de Raymond Kévorkian, infra, pp. 75-85. première étincelle. En ce sens, une mise à plat de la controverse qui a divisé les historiens peut s'avérer utile. L'un de ses principaux enseignements, qu'il faut souligner, tient précisément au caractère pluriel de la profession historienne, qui n'entretient, en France, aucun consensus à ce sujet, loin de l'image d'unanimisme et de corporatisme qui semble s'être diffusée auprès du grand public. Les termes du débat n’ont pas toujours aidé à le rendre lisible, comme le montre l'impasse à laquelle a mené, depuis 2005, l'usage abusif et tendancieux d'une notion de « lois mémorielles » qui n'a, à ce jour, fait l'objet d'aucune définition rigoureuse. Le tempo imposé au débat par les logiques et les échéances politico-médiatiques, ne contribue pas lui non plus à son approfondissement. Il n'y a quà voir les circonstances et la méthode qui ont présidé l'hiver dernier, avec la bénédiction de l'Élysée, à la relance d'une loi contre la négation des génocides ${ }^{63}$. Cette question complexe est toujours en atten- 
te d'un débat ouvert et serein, qui permette véritablement d'éclairer les citoyens sur leurs choix, et dans lequel les historiens ont toute leur place. 